\title{
3D POISSON MICROSCOPY DECONVOLUTION WITH HESSIAN SCHATTEN-NORM REGULARIZATION
}

\author{
Stamatios Lefkimmiatis and Michael Unser \\ Biomedical Imaging Group, EPFL, CH-1015 Lausanne, Switzerland \\ Email: [stamatis.lefkimmiatis,michael.unser] depfl.ch
}

\begin{abstract}
Inverse problems with shot noise arise in many modern biomedical imaging applications. The main challenge is to obtain an estimate of the underlying specimen from measurements corrupted by Poisson noise. In this work, we propose an efficient framework for photonlimited image reconstruction, under a regularization approach that relies on matrix-valued operators. Our regularizers involve the Hessian operator and its eigenvalues. They are second-order regularizers that are well suited to biomedical images. For the solution of the arising minimization problem, we propose an optimization algorithm based on an augmented-Lagrangian formulation and specifically tailored to the Poisson nature of the noise. To assess the quality of the reconstruction, we provide experimental results on 3D image stacks of biological images for microscopy deconvolution.
\end{abstract}

Index Terms - Poisson noise, photon-limited imaging, Hessian operator, Schatten norms, ADMM.

\section{INTRODUCTION}

Fluorescense microscopy is a valuable tool for biologists. It allows them to observe and study the structure of specimens but faces two basic limitations. The first limitation is the presence of an outof-focus blur which reduces the resolution of the acquired image stacks and the ability to clearly distinguish fine structures. This blur is an inherent limitation encountered in every diffraction-limited optical imaging system. The second limitation is the measurement noise which further degrades the quality of the recorded images. In many cases, due to practical constraints such as short exposure-time or photo-toxicity, the acquisition is performed under low-light conditions. Then, the most dominant source of noise is quantum (or shot) noise [1], which is signal-dependent and obeys a Poisson distribution. Together, these two limitations can have a severe negative impact on biological studies.

To mitigate the degradations due to the optics of the acquisition system and to attenuate the measurement noise, Poisson 3D image restoration can serve as a pre-processing technique.

\subsection{Prior Work}

Many reconstruction algorithms have been proposed for solving Poisson inverse problems. Most of these methods can be interpreted as optimization techniques of an objective function. For instance, the Richardson-Lucy (RL) algorithm [2,3] corresponds to computing a Poisson maximum likelihood (ML) estimate. Another approach, which is widely followed, is to obtain the solution as a penalized ML estimate. In this case, one of the most popular penalties is based

This work was supported (in part) by the Hasler Foundation and the Indo-Swiss Joint Research Program. on the total variation (TV) semi-norm [4] and an efficient algorithm for computing the solution has been recently proposed [5]. TV produces good estimates for a variety of imaging applications. However, it also promotes piecewise-constant solutions which can lead to an oversharpening of the image contours. This is known as staircase effect and can be a serious drawback in biomedical applications.

Having in mind that biomedical images consist mostly of ridges and filament-like structures, which can be modeled by piecewisesmooth intensity variations, second-order regularizers seems to be more appropriate than TV and can eliminate the staircase effect. This motivated us to recently introduce a family of non-quadratic secondorder regularizers [6, 7]. They are based on the Hessian eigenvalues and can be considered as second-order extensions of TV, in the sense that they satisfy exactly the same invariance properties while involving second-order derivatives instead of first-order ones. Their main feature is that they favor piecewise-smooth solutions, as opposed to TV which favors piecewise-constant ones. Therefore, for the type of images met in biomedical imaging applications, second-order regularization provides reconstructions that better approximate intensity variations. Currently our regularizers are combined efficiently with a quadratic fidelity term, which is appropriate only for Gaussian noise.

\subsection{Contributions}

The contributions of this work are twofold:

- We introduce a family of derivative-based regularizers for 3D photon-limited image reconstruction. These are second-order non-quadratic regularizers and depend on the eigenvalues of the Hessian operator computed at every voxel of the image volume.

- We lift the restriction of a quadratic data term and propose an optimization algorithm which is specifically tailored to the Poisson nature of the noise. Our proposed algorithm is based on an augmented-Lagrangian framework and follows the same principles as other existing algorithms in the literature (see [5], for instance). However, while the latter are designed to work with regularizers involving scalar and vectorialvalued operators, ours is designed to handle matrix-valued linear operators. This generalization is important since it introduces additional difficulties which cannot be handled in a trivial way. Moreover, the splitting approach we employ is novel and reduces the number of required auxiliary variables.

The paper is organized as follows: In Section 2, we formulate the problem and introduce our regularizers. In Section 3, we describe our proposed 3D Poisson restoration algorithm. Finally, in Section 4, we perform deconvolution experiments on 3D biomedical images to assess the performance of our approach. 


\section{POISSON INVERSE PROBLEMS}

\subsection{Problem Formulation}

Our approach for reconstructing the underlying intensities from the measurements is based on the image-formation model

$$
\mathbf{y}=\mathcal{T}(\mathbf{K} \mathbf{x}+\mathbf{b})
$$

where $\mathbf{K} \in \mathbb{R}^{M \times N}$ is a matrix that models the spatial response of the imaging device, for instance the point spread function (psf) of the microscope, $\mathbf{b} \in \mathbb{R}_{+}^{M}$ is constant and models the background intensity of the image, $\mathcal{T}$ is a degradation operator that models the measurement noise, and $\mathbf{y} \in \mathbb{R}_{+}^{M}, \mathbf{x} \in \mathbb{R}_{+}^{N}$ are the vectorized versions of the observed image and the image to be estimated, respectively. Both $\mathbf{x}$ and $\mathbf{y}$ are assumed to be non negative valued. We further expand (1) by noting that the unobserved intensities $\mathbf{x}$ of the underlying image are related to other photon intensities $\boldsymbol{\lambda}$ (which are also unobserved) through the relation $\boldsymbol{\lambda}=\mathbf{K} \mathbf{x}+\mathbf{b}$. For the elements of $\boldsymbol{\lambda}$ to be interpreted as photon intensities, we need to make the hypothesis that $\boldsymbol{\lambda} \in \mathbb{R}_{+}^{M}$. This hypothesis is consistent with the idea that $\mathbf{K}$ represents a linear degradation effect, such as blur.

Under the basic photon-limited imaging model, the measurement noise implied by $\mathcal{T}$ in (1) is signal-dependent. In particular, the observed image $\mathbf{y}$ can be considered as a vector containing the samples of a random sequence $\mathbf{Y}=\left(Y_{1}, \ldots, Y_{M}\right)$. The $M$ random variables $Y_{m}$ are conditionally independent upon $\boldsymbol{\lambda}$, and each one follows a Poisson distribution with rate parameter $\lambda_{m}$, so that $Y_{m} \sim$ Pois $\left(Y_{m} \mid \lambda_{m}\right)$. The likelihood of $\mathbf{x}$ is expressed as

$$
p(\mathbf{Y}=\mathbf{y} \mid \mathbf{x})=\prod_{m=1}^{M} \frac{e^{-[\mathbf{K} \mathbf{x}+\mathbf{b}]_{m}}[\mathbf{K} \mathbf{x}+\mathbf{b}]_{m}^{y_{m}}}{y_{m} !},
$$

where $[\cdot]_{m}$ indicates the $m$ th element of the vector argument.

One standard approach to recover $\mathbf{x}$ from the measurements $\mathbf{y}$ is to minimize the negative log-likelihood

$$
f_{L}(\mathbf{x})=\mathbf{1}^{T}(\mathbf{K} \mathbf{x}+\mathbf{b})-\mathbf{y}^{T} \log (\mathbf{K} \mathbf{x}+\mathbf{b})+\iota_{\mathcal{C}}(\mathbf{x}),
$$

where 1 is a vector of size $M$ whose elements are set to 1 and $\iota_{\mathcal{C}}$ is the indicator function of the convex set $\mathcal{C}=\mathbb{R}_{+}^{N}$ that represents the non negativity constraints on the solution. The convention is that $\iota_{\mathcal{C}}(\mathbf{x})$ takes values 0 for $\mathbf{x} \in \mathcal{C}$ and $\infty$ otherwise. In order for (3) to be consistent with (2), the convention that $0 \log (0)=0$ is also used. The minimizer of (3) is equivalent to the ML estimate. One way to obtain it is by using the RL algorithm, which is an iterative multiplicative gradient-type technique [2,3]. Unfortunately, the ill-posed nature of the problem implies that the operator $\mathbf{K}$ for the cases of practical interest is either ill-conditioned or singular, and the corresponding ML estimator has a large variance. For this reason, the RL algorithm should be terminated early in practice, just after few iterations. Otherwise, the noise gets amplified and results in an estimate dominated by noise.

To alleviate this amplification of the noise, one can instead minimize the penalized version of the negative log-likelihood given by:

$$
f(\mathbf{x})=f_{L}(\mathbf{x})+\phi(\mathbf{x}),
$$

where the role of $\phi$ is to constrain the set of plausible solutions. The minimizer of (4) leads to a maximum a posteriori (MAP) estimate, with $\phi(\mathbf{x})$ being interpreted as the negative logarithm of the prior distribution of the underlying image $\mathbf{x}$. Note that the minimization of (4) also arises from a variational viewpoint, with (4) considered as an energy functional consisting of two terms: the data fidelity $f_{L}$ and the regularizer $\phi$.

\subsection{Regularization Approach}

Our Hessian Schatten-norm regularizers are defined as [7]

$$
\mathcal{H S}_{p}(\mathbf{x})=\|\mathcal{H} \mathbf{x}\|_{1, p}=\sum_{n=1}^{N}\left\|[\mathcal{H} \mathbf{x}]_{n}\right\|_{\mathcal{S}_{p}}, \forall p \geq 1
$$

where

$$
[\mathcal{H} \mathbf{x}]_{n}=\left[\begin{array}{ccc}
{\left[\Delta_{r_{1} r_{1}} \mathbf{x}\right]_{n}} & {\left[\Delta_{r_{2} r_{1}} \mathbf{x}\right]_{n}} & {\left[\Delta_{r_{3} r_{1}} \mathbf{x}\right]_{n}} \\
{\left[\Delta_{r_{1} r_{2}} \mathbf{x}\right]_{n}} & {\left[\Delta_{r_{2} r_{2}} \mathbf{x}\right]_{n}} & {\left[\Delta_{r_{3} r_{2}} \mathbf{x}\right]_{n}} \\
{\left[\Delta_{r_{1} r_{3}} \mathbf{x}\right]_{n}} & {\left[\Delta_{r_{2} r_{3}} \mathbf{x}\right]_{n}} & {\left[\Delta_{r_{3} r_{3}} \mathbf{x}\right]_{n}}
\end{array}\right]
$$

is the discrete Hessian and $\left[\Delta_{r_{i} r_{j}} \mathbf{x}\right]_{n}$ denotes the discrete approximations of the second-order partial derivatives along two dimensions of the image (volume) at voxel $n$. In (5), $\|\mathbf{X}\|_{\mathcal{S}_{p}}$ denotes the Schatten norm of order $\mathrm{p}$ of a matrix $\mathbf{X} \in \mathbb{R}^{K \times K}$, defined as

$$
\|\mathbf{X}\|_{\mathcal{S}_{p}}=\left(\sum_{k=1}^{K}\left|\sigma_{k}\right|^{p}\right)^{\frac{1}{p}}
$$

where $\sigma_{k}$ corresponds to the $k$ th eigenvalue of $\mathbf{X}$. Definition (5) reveals a close relation between our regularizers and the sparsitypromoting group norms commonly met in compressive sensing (see [8], for instance). However, an important and non-trivial difference is that, in (5), the mixed norm is a vector-matrix norm rather than a vector-vector norm. Therefore, the sparsity is enforced on the eigenvalues of the Hessian rather than directly on its elements.

\section{PROPOSED MINIMIZATION STRATEGY}

Based on the photon-limited imaging model (1) and under our Hessian Schatten-norm regularization (5), a penalized ML estimate $\hat{\mathbf{x}}$ of the underlying image is obtained as the minimizer

$$
\begin{aligned}
\hat{\mathbf{x}}=\underset{\mathbf{x} \in \mathbb{R}^{N}}{\arg \min }\left(f(\mathbf{x}) \triangleq \mathbf{1}^{T}(\mathbf{K} \mathbf{x}+\mathbf{b})-\mathbf{y}^{T} \log (\mathbf{K} \mathbf{x}+\mathbf{b})\right. \\
\left.+\tau\|\mathcal{H} \mathbf{x}\|_{1, p}+\iota_{\mathcal{C}}(\mathbf{x})\right),
\end{aligned}
$$

where $\tau \geq 0$ is the regularization parameter which balances the influence of the data fidelity and the penalty term. The form of (8) is difficult to work with due to the coupling that exists among the fidelity term and the regularizer. Another difficulty stems from the fact that our regularizers involve matrix-valued (Hessian) instead of vector-valued operators such as the gradient used in TV.

Next, we describe a novel algorithm which overcomes these difficulties. To remove the dependency among the fidelity term and the regularizer, we introduce the auxiliary variables $\mathbf{z}_{1}=\mathbf{K} \mathbf{x}+\mathbf{b}$, $\mathbf{z}_{2}=\mathbf{x}$ and express (8) in the equivalent constrained form

$$
\min _{\substack{\mathbf{x}, \mathbf{z}_{2} \in \mathbb{R}^{N} \\ \mathbf{z}_{1} \in \mathbb{R}^{M}}} \underbrace{\mathbf{1}^{T} \mathbf{z}_{1}-\mathbf{y}^{T} \log \mathbf{z}_{1}}_{f_{1}\left(\mathbf{z}_{1}\right)}+\underbrace{\left\|\boldsymbol{\mathcal { H }} \mathbf{z}_{2}\right\|_{1, p}+\iota_{\mathcal{C}}\left(\mathbf{z}_{2}\right)}_{f_{2}\left(\mathbf{z}_{2}\right)} .
$$

Our splitting approach differs from the one used in [5], reducing the number of auxiliary variables from three to two. Since (9) corresponds to a constrained minimization problem, we solve it by forming the augmented Lagrangian and employing the ADMM algorithm [9]. We recall that ADMM solves problems of the general form

$$
\min _{\mathbf{A} \mathbf{x}+\mathbf{B} \mathbf{z}=\mathbf{c}} g(\mathbf{x})+f(\mathbf{z})
$$


Algorithm 1 : Hessian Schatten-norm Poisson image reconstruction by augmented Lagrangian (HSPIRAL).

Input: $\mathbf{y}, \mathbf{K}, \mathbf{b}, \tau>0, \alpha>0, p \geq 1$,

$\mathcal{P}_{\mathcal{D}}$ (Projection operator onto the convex set $\mathcal{D}$ ).

Initialization: $\mathbf{x}^{0}=\mathbf{y}, \mathbf{s}_{1}^{0}=\mathbf{0}, \mathbf{s}_{2}^{0}=\mathbf{0}$.

Output: $\hat{\mathbf{x}}$ (solution of $(8)$ ).

while stopping criterion is not satisfied do

$$
\begin{aligned}
& \mathbf{u}_{1}^{t} \leftarrow \mathbf{K} \mathbf{x}^{t}+\mathbf{b}+\mathbf{s}_{1}^{t} ; \\
& \mathbf{u}_{2}^{t} \leftarrow \mathbf{x}^{t}+\mathbf{s}_{2}^{t} ; \\
& \mathbf{z}_{1}^{t+1} \leftarrow \frac{1}{2}\left(\mathbf{u}_{1}^{t}-\frac{1}{\alpha}+\sqrt{\left(\mathbf{u}_{1}^{t}-\frac{1}{\alpha}\right)^{2}+\frac{4 \mathbf{y}}{\alpha}}\right) ; \\
& \mathbf{z}_{2}^{t+1} \leftarrow \underset{\mathbf{z}_{2} \in \mathbb{R}^{n}}{\arg \min } \frac{1}{2}\left\|\mathbf{z}_{2}-\mathbf{u}_{2}^{t}\right\|_{2}^{2}+\frac{\tau}{\alpha}\left\|\mathcal{H} \mathbf{z}_{2}\right\|_{1, p}+\iota_{\mathcal{D}}\left(\mathbf{z}_{2}\right) ; \\
& \mathbf{v}^{t} \leftarrow \mathbf{K}^{T}\left(\mathbf{z}_{1}^{t+1}-\mathbf{b}-\mathbf{s}_{1}^{t}\right)+\left(\mathbf{z}_{2}^{t+1}-\mathbf{s}_{2}^{t}\right) ; \\
& \mathbf{x}^{t+1} \leftarrow\left(\mathbf{K}^{T} \mathbf{K}+\mathbf{I}\right)^{-1} \mathbf{v}^{t} ; \\
& \mathbf{s}_{1}^{t+1} \leftarrow \mathbf{s}_{1}^{t}+\mathbf{K} \mathbf{x}^{t+1}+\mathbf{b}-\mathbf{z}_{1}^{t+1} ; \\
& \mathbf{s}_{2}^{t+1} \leftarrow \mathbf{s}_{2}^{t}+\mathbf{x}^{t+1}-\mathbf{z}_{2}^{t+1} ; \\
& t \leftarrow t+1 ;
\end{aligned}
$$

end

return $x^{t}$

where $\mathbf{x} \in \mathbb{R}^{N}, \mathbf{z} \in \mathbb{R}^{M}, \mathbf{A} \in \mathbb{R}^{L \times N}, \mathbf{B} \in \mathbb{R}^{L \times M}$, and $\mathbf{c} \in \mathbb{R}^{L}$. This is compatible with the form of Problem (9) and can be verified by setting in (10) $\mathbf{z}=\left(\mathbf{z}_{1}, \mathbf{z}_{2}\right), \mathbf{A}=\left(\mathbf{K}, \mathbf{I}_{N}\right), \mathbf{B}=-\mathbf{I}_{M+N}$, $\mathbf{c}=(-\mathbf{b}, \mathbf{0}), g(\mathbf{x})=0$ and $f(\mathbf{z})=f_{1}\left(\mathbf{z}_{1}\right)+f_{2}\left(\mathbf{z}_{2}\right)$.

The augmented Lagrangian for Problem (9) is

$$
\mathcal{L}_{\alpha}(\mathbf{x}, \mathbf{z}, \boldsymbol{\eta})=f(\mathbf{z})+\langle\boldsymbol{\eta}, \mathbf{A x}-\mathbf{z}-\mathbf{c}\rangle+\frac{\alpha}{2}\|\mathbf{A} \mathbf{x}-\mathbf{z}-\mathbf{c}\|_{2}^{2},
$$

where $\boldsymbol{\eta}=\left(\boldsymbol{\eta}_{1}, \boldsymbol{\eta}_{2}\right) \in \mathbb{R}^{M+N}$ corresponds to the dual variables (Lagrange multipliers) and $\alpha>0$ is a penalty parameter. The ADMM iterations that solve (9) are given by

$$
\begin{aligned}
& \mathbf{z}^{t+1}=\underset{\mathbf{z} \in \mathbb{R}^{M+N}}{\arg \min } f(\mathbf{z})+\frac{\alpha}{2}\left\|\mathbf{z}-\left(\mathbf{A} \mathbf{x}^{t}-\mathbf{c}+\mathbf{s}^{t}\right)\right\|_{2}^{2} \\
& \mathbf{x}^{t+1}=\underset{\mathbf{x} \in \mathbb{R}^{n}}{\arg \min }\left\|\mathbf{A} \mathbf{x}-\left(\mathbf{z}^{t+1}+\mathbf{c}-\mathbf{s}^{t}\right)\right\|_{2}^{2} \\
& \mathbf{s}^{t+1}=\mathbf{s}^{t}+\mathbf{A} \mathbf{x}^{t+1}-\mathbf{c}-\mathbf{z}^{t+1},
\end{aligned}
$$

\begin{tabular}{|c|c|c|c|c|c|c|c|}
\hline \multirow{2}{*}{ Data } & \multirow{2}{*}{$\begin{array}{l}\text { Avg. Photon } \\
\text { Intensity }\end{array}$} & \multicolumn{6}{|c|}{ Methods (PSNR) } \\
\hline & & Blurred & $\mathrm{RL}$ & GTK & TV & $\mathcal{H S}_{2}$ & $\mathcal{H S}_{1}$ \\
\hline \multirow{2}{*}{ Stack A } & 10 & 20.97 & 24.23 & 25.33 & 25.87 & 25.99 & 26.05 \\
\hline & 50 & 21.90 & 26.69 & 26.27 & 27.21 & 27.33 & 27.40 \\
\hline \multirow{2}{*}{ Stack B } & 10 & 20.65 & 22.66 & 22.99 & 23.37 & 23.44 & 23.48 \\
\hline & 50 & 21.16 & 24.02 & 23.35 & 24.38 & 24.46 & 24.48 \\
\hline
\end{tabular}

where $\mathbf{s}=\boldsymbol{\eta} / \alpha$ is the scaled version of the dual variables. The only remaining task is the solution of the subproblems appearing in (12).

For the z-update of the ADMM, we observe that the argument in (12a) is decoupled in 2 independent components. Indeed,

$$
\begin{aligned}
f(\mathbf{z})+ & \frac{\alpha}{2}\left\|\mathbf{z}-\left(\mathbf{A} \mathbf{x}^{t}-\mathbf{c}+\mathbf{s}^{t}\right)\right\|_{2}^{2} \\
& =\sum_{k=1}^{2} f_{k}\left(\mathbf{z}_{k}\right)+\frac{\alpha}{2}\left\|\mathbf{z}_{k}-\left(\mathbf{A}_{k} \mathbf{x}^{t}-\mathbf{c}_{k}+\mathbf{s}_{k}^{t}\right)\right\|_{2}^{2},
\end{aligned}
$$

thus we can compute each $\mathbf{z}_{k}^{t+1}$ independently as

$$
\mathbf{z}_{k}^{t+1}=\operatorname{prox}_{f_{k} / \alpha}\left(\mathbf{u}_{k}^{t}\right), \forall k=1,2,
$$

where $\operatorname{prox}_{f}(\mathbf{y})$ is the Moreau proximity operator [10] of a function $f$ evaluated at $\mathbf{y}$ and $\mathbf{u}_{k}^{t}=\mathbf{A}_{k} \mathbf{x}^{t}-\mathbf{c}_{k}+\mathbf{s}_{k}^{t}$. The proximal
Table 1. PSNR comparisons on 3D Poisson image deconvolution.

$\operatorname{map}_{\operatorname{prox}} \operatorname{fr}_{1 / \alpha}\left(\mathbf{u}_{1}^{t}\right)$, with $\mathbf{u}_{1}^{t}=\mathbf{K} \mathbf{x}^{t}+\mathbf{b}+\mathbf{s}_{1}^{t}$, is separable and is computed component-wise. The solution for each component of the auxiliary variable $\mathbf{z}_{1}$ is standard and corresponds to finding the positive root of a second-order polynomial. Based on this, we have

$$
\mathbf{z}_{1}^{t+1}=\frac{1}{2}\left(\mathbf{u}_{1}^{t}-\frac{1}{\alpha}+\sqrt{\left(\mathbf{u}_{1}^{t}-\frac{1}{\alpha}\right)^{2}+\frac{4 \mathbf{y}}{\alpha}}\right),
$$

where all the operations are performed component-wise. The non standard part of our formulation is the evaluation of the proximal $\operatorname{map}_{\operatorname{prox}} f_{2 / \alpha}\left(\mathbf{u}_{2}^{t}\right)$, with $\mathbf{u}_{2}^{t}=\mathbf{x}^{t}+\mathbf{s}_{2}^{t}$. To obtain it, we employ the iterative algorithm that is fully described in our recent paper [7]. This method finds efficiently a numerical solution to the problem

$$
\underset{\mathbf{x} \in \mathbb{R}^{N}}{\arg \min } \frac{1}{2}\|\mathbf{x}-\mathbf{u}\|_{2}^{2}+\tau\|\mathcal{H} \mathbf{x}\|_{1, p}+\iota_{\mathcal{D}}(\mathbf{x}) \forall p \geq 1,
$$

where $\mathcal{D}$ is a convex set. (In our case, $\mathcal{D} \equiv \mathcal{C}$ ).

The $\mathrm{x}$-update $(12 \mathrm{~b})$ corresponds to solving a quadratic minimization problem whose formal solution is given by

$$
\begin{aligned}
\mathbf{x}^{t+1} & =\left(\mathbf{A}^{T} \mathbf{A}\right)^{-1} \mathbf{A}^{T}\left(\mathbf{z}^{t+1}+\mathbf{c}-\mathbf{s}^{t}\right) \\
& =\left(\mathbf{K}^{T} \mathbf{K}+\mathbf{I}\right)^{-1}\left(\mathbf{K}^{T}\left(\mathbf{z}_{1}^{t+1}-\mathbf{b}-\mathbf{s}_{1}^{t}\right)+\left(\mathbf{z}_{2}^{t+1}-\mathbf{s}_{2}^{t}\right)\right),
\end{aligned}
$$

where $(\cdot)^{T}$ stands for the adjoint operator. Assuming periodic boundary conditions for $\mathbf{x}$, the matrix $\left(\mathbf{K}^{T} \mathbf{K}+\mathbf{I}\right)^{-1}$ is diagonalized by a fast Fourier transform and the solution is computed efficiently in the Fourier domain. Similarly to the z-update, the s-update in (12c) is performed in a decoupled manner as

$$
\begin{aligned}
& \mathbf{s}_{1}^{t+1}=\mathbf{s}_{1}^{t}+\mathbf{K} \mathbf{x}^{t+1}+\mathbf{b}-\mathbf{z}_{1}^{t+1}, \mathbf{s}_{1} \in \mathbb{R}^{M} \\
& \mathbf{s}_{2}^{t+1}=\mathbf{s}_{2}^{t}+\mathbf{x}^{t+1}-\mathbf{z}_{2}^{t+1}, \mathbf{s}_{2} \in \mathbb{R}^{N} .
\end{aligned}
$$

A summary of the overall proposed numerical algorithm, named HSPIRAL (Hessian Schatten-norm Poisson image reconstruction by augmented Lagrangian), is provided in Algorithm 1.

\section{EXPERIMENTS}

To assess the performance of our Hessian Schatten-norm regularization framework, we report results for the task of 3D deconvolution, using two clean confocal image stacks as ground truth and simulating widefield acquisition with Poisson noise. We compare the results we obtained using two of our regularizers, for $p=1,2$, with three alternative approaches, namely the RL algorithm, TV, and gradient-based Tikhonov regularization (GTK). In our simulations, we use a $3 \mathrm{D}$ psf produced by our software, which is freely available at http://bigwww.epfl.ch/algorithms/psfgenerator/, according to the Gibson-Lani PSF model. In Table 1, we provide numerical results in terms of PSNR for two different levels of Poisson noise. Since Poisson noise is signal-dependent, to simulate the different noise levels we multiply the original confocal image stacks by the appropriate gain that results in an average number of photons 

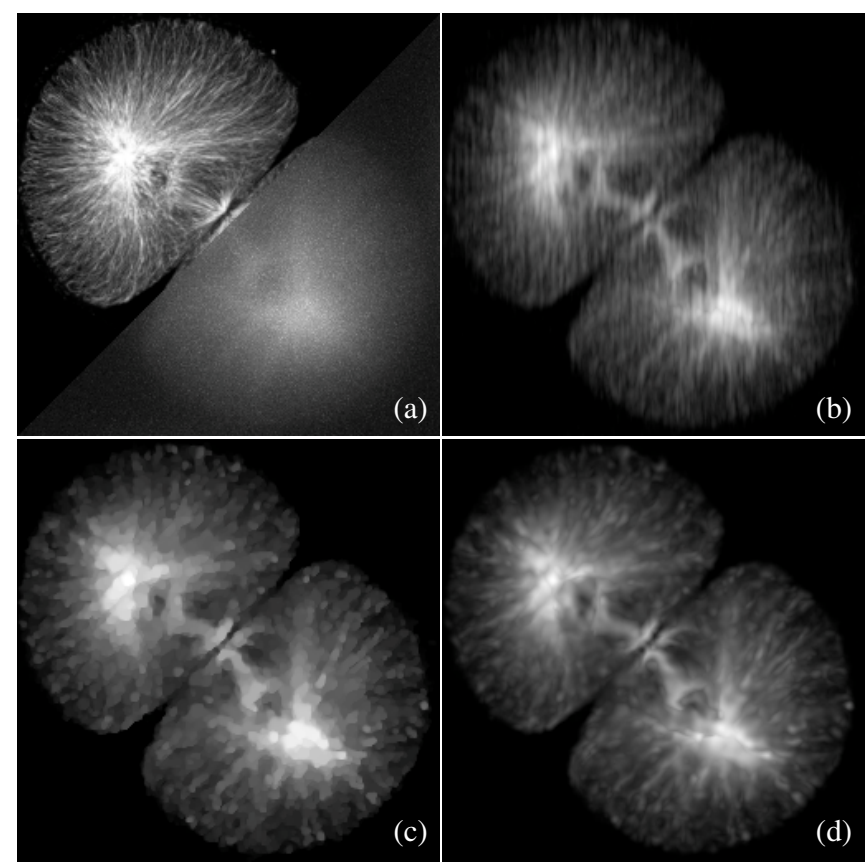

Fig. 1. 3D Poisson-deconvolution results on a stack of microtubules (Stack A) of size $256 \times 256 \times 52$. Maximum-intensity projection (along the z-axis) of the (a) ground-truth (upper-left) and blurred (lower-right) stack (PSNR=20.97 dB), (b) GTK (PSNR=25.33 dB), (c) TV (PSNR=25.87 dB), (d) $\mathcal{H S}_{2}(\mathrm{PSNR}=25.99 \mathrm{~dB})$.

at every voxel of $(10,50)$. For the sake of fairness, the results reported for every regularizer under comparison are obtained using the individualized regularization parameter $\tau$ that gives the best PSNR performance. For the RL algorithm, we report the iteration that produces the highest PSNR score.

From Table 1, we observe that our regularizers provide the best PSNR scores, with the $\mathcal{H} \mathcal{S}_{1}$ penalty achieving a slightly better performance than the $\mathcal{H S}_{2}$. Besides the quantitive comparisons and the seemingly small PSNR improvement over TV, the efficacy of our proposed framework can also be visually appreciated from the representative deconvolution examples shown in Figs. 1 and 2. In these examples, we verify that our regularizers restore in a better way the fine structures of the specimens without introducing severe artifacts. On the other hand, the RL algorithm does not remove the noise efficiently, the GTK suffers from ringing artifacts, while TV tends to oversmooth the images. This tendency is responsible for shuffling details of the image and broadening its fine structures.

\section{CONCLUSIONS}

We proposed a framework for 3D Poisson image restoration subject to second-order derivative-based regularization. The employed regularizers depend on the eigenvalues of the Hessian operator. We showed experimentally that these functionals are well suited to biomedical images and provide better reconstruction results than other popular deblurring techniques. We designed a novel and efficient optimization algorithm for the minimization of the corresponding objective functions. Our algorithm is based on an augmented Lagrangian formulation and can effectively deal with the high dimensionality of the data.

Acknowledgments: The authors would like to thank George von Dassow for kindly providing the confocal stacks used in Figs. 1-2.
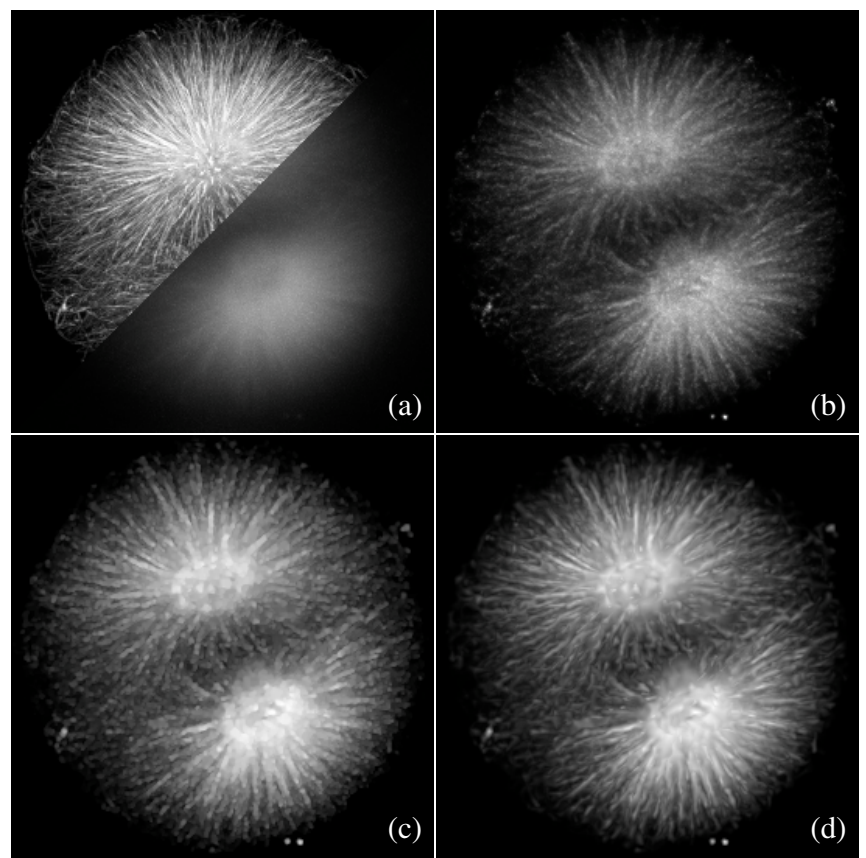

Fig. 2. 3D Poisson-deconvolution results on a stack of microtubules (Stack B) of size $272 \times 272 \times 72$. Maximum-intensity projection (along the z-axis) of the (a) ground-truth (upper-left) and blurred (lower-right) stack (PSNR=21.16 dB), (b) RL (PSNR=24.02 dB), (c) $\mathrm{TV}(\mathrm{PSNR}=24.38 \mathrm{~dB}),(\mathrm{d}) \mathcal{H S}_{1}(\mathrm{PSNR}=24.48 \mathrm{~dB})$.

\section{REFERENCES}

[1] H. Barett, "Objective assessment of image quality: effects of quantum noise and object variability," J. Opt. Soc. Am. A, vol. 7, no. 7, pp. 1266-1278, 1990.

[2] W. H. Richardson, "Bayesian-based iterative method of image restoration," J. Opt. Soc. Am. A, vol. 62, no. 1, pp. 55-59, 1972.

[3] L. B. Lucy, "An iterative technique for the rectification of observed distributions," The astronomical journal, vol. 79, pp. 745, 1974.

[4] L. Rudin, S. Osher, and E. Fatemi, "Nonlinear total variation based noise removal algorithms," Physica D, vol. 60, pp. 259-268, 1992.

[5] M.A.T. Figueiredo and J.M. Bioucas-Dias, "Restoration of Poissonian images using alternating direction optimization," IEEE Trans. Image Processing, vol. 19, no. 12, pp. 3133-3145, 2010.

[6] S. Lefkimmiatis, A. Bourquard, and M. Unser, "Hessian-based norm regularization for image restoration with biomedical applications," IEEE Trans. Image Processing, vol. 21, no. 3, pp. 983-995, 2012.

[7] S. Lefkimmiatis, J. Ward, and M. Unser, "Hessian Schatten-norm regularization for linear inverse problems," IEEE Trans. Image Processing (to appear), 2013, doi:10.1109/TIP.2013.2237919.

[8] F. Bach, R. Jenatton, J. Mairal, and G. Obozinski, "Optimization with sparsity-inducing penalties," Arxiv preprint arXiv:1108.0775, 2011.

[9] S. Boyd, N. Parikh, E. Chu, B. Peleato, and J. Eckstein, Distributed Optimization and Statistical Learning via the Alternating Direction Method of Multipliers, Now Publishers, 2011.

[10] P. L. Combettes and V. R. Wajs, "Signal recovery by proximal forwardbackward splitting," Multiscale Model. Simul., vol. 4, no. 4, pp. 11681200, 2005. 